EOMmun Communication et organisation

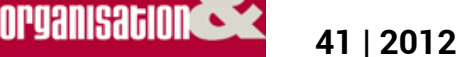

La mutation du métier de communicant public

\title{
La mutation du métier de communicant public
}

Annick Monseigne et Geneviève Guilhaume

\section{OpenEdition}

Journals

Édition électronique

URL : http://journals.openedition.org/communicationorganisation/3694

DOI : 10.4000/communicationorganisation.3694

ISSN : $1775-3546$

Éditeur

Presses universitaires de Bordeaux

Édition imprimée

Date de publication : 1 juin 2012

Pagination : 5-11

ISBN : $978-2-86781-821-9$

ISSN : 1168-5549

Référence électronique

Annick Monseigne et Geneviève Guilhaume, "La mutation du métier de communicant public », Communication et organisation [En ligne], 41 | 2012, mis en ligne le 29 mars 2013, consulté le 10 décembre 2020. URL : http://journals.openedition.org/communicationorganisation/3694 ; DOI : https://doi.org/10.4000/communicationorganisation.3694 


\title{
La mutation du métier de communicant public
}

\author{
Annick Monseigne', Geneviève Guilhaume ${ }^{2}$
}

Le métier de communicant, et plus particulièrement de communicant public, a connu en ce début de siècle de profonds bouleversements. Bouleversements repérés à l'échelle planétaire avec la révolution numérique et l'avènement du web collaboratif (Bouquillon et Matthews, 2010), mais aussi à l'échelle locale avec un changement paradigmatique de la communication publique favorisant les pratiques managériales et la prégnance des dispositifs délibératifs (Blondiaux, Sintomer, 2002). Fin d'un modèle de communication exclusivement unidirectionnel, politiques publiques réformées, temporalités bouleversées, injonction à la participation, impératifs gestionnaires du nouveau management public, émergence de nouveaux métiers de la communication, constituent des changements de fond qui bousculent la réalité des institutions communicantes, redéfinissent leurs logiques organisationnelles jusqu'ici liées à une communication normative de l'information réglementaire et une communication de promotion institutionnelle et/ou politique classique.

Pour autant, traiter de la mutation du métier de communicant public est une gageure tant la problématique est complexe.

Complexité identifiée à travers deux grands défis sociétaux aux logiques opposées. Le premier, de nature exogène, d'ordre technologique, est né de l'explosion des médias sociaux. Il implique pour l'organisation publique de s'adapter au changement en créant et en gérant ses propres processus de mise en relation sur le web. Plus endogène, le second, d'ordre organisationnel, a puisé sa motivation dans le changement paradigmatique de la communication publique. Sa mission est d'assurer le passage d'une communication bureaucratique (Weber, 1921) à une communication managériale soumise

1. Maître de conférences en Sciences de I'Information et de la Communication à I'IUT Michel de Montaigne-Université Bordeaux 3. Chercheur au laboratoire Médiation, Information, Communication, Art (MICA), axe Communication, Organisations et Sociétés, elle compte à son actif vingt ans d'expérience de terrain comme consultante en communication auprès des collectivités territoriales; annick.monseigne@free.fr 2. Maître de conférences en Sciences de l'Information et de la Communication à l'Université Bordeaux 3. Elle est chercheur au laboratoire Médiation, Information, Communication, Art (MICA), axe Communication, Organisations et Sociétés et responsable du master Communication publique et politique; genevieve. guilhaume@u-bordeaux3.fr 
aux logiques d'efficacité et de résultat, d'une communication unilatérale à une communication plus inclusive sommée d'intégrer les nouvelles normes délibératives (Habermas, 1992).

Témoins d'une période de transition majeure, nous assistons aux balbutiements d'une nouvelle ère de la politique publique. Toujours confrontés au dilemme du partage entre ce que Max Weber (1919) nomme l'éthique de conviction et l'éthique de responsabilité, les élus de la République voient, aujourd'hui, leurs responsabilités s'accroître au fur et à mesure du désengagement de l'Etat et des transferts de compétence de l'Etat vers les collectivités territoriales. Les communicants qui travaillent en tandem avec les élus, évoluent, dès lors, dans un nouveau cadre politique d'évaluation de la performance pour mieux répondre aux exigences des logiques de résultat venues compléter les logiques de moyens engagées au nom du service public. Ce contexte de crise généralisée leur commande de composer avec un lot d'exigences et de pressions les conduisant tant sur les voies de la rationalisation du travail et des valeurs de productivité que de la créativité (Boltanski et Thévenot, 1991). "Nouvel esprit du capitalisme » (Boltanski et Chapiello, 1999) et esprit nouvelle gouvernance semblent résolument imbriqués.

Les faits sont là pour nous rappeler la difficile conciliation entre contrôle et ouverture, verticalité et horizontalité, discours et conversations, pratiques top down et pratiques bottom up. Il faut, à cet égard, préciser l'ampleur des problématiques auxquelles sont confrontés les communicants publics. La bataille est rude sur tous les fronts, qu'il s'agisse :

- des évolutions technologiques majeures à affronter, comme celle de la diffusion des nouvelles formes de communication sur les réseaux sociaux. Temporalités éclatées, canaux multipliés, flux continus, partage des contenus, le web 2.0, dit collaboratif, bouleverse l'univers normé et normatif des institutions publiques.

- des réformes de structure au niveau local notamment avec les mouvements incessants de la décentralisation-mutualisation, induits tant par les impératifs du renouveau démocratique (dispositifs participatifs) que par des contraintes économiques (budgets publics).

- des réformes du management, dans le cadre de la communauté économique européenne, le Nouveau Management Public imposant des impératifs de résultats proches des logiques entrepreneuriales dans un secteur à forte identité de service public. En effet, les modes de gestion des organisations publiques, bureaucratiques sont aujourd'hui largement orientés par le contrôle sur les résultats. Ces organisations sont soumises à une concurrence accrue, liée au principe de subsidiarité, reconfiguré selon les périodes, l'action publique elle-même n'étant plus le monopole des organisations publiques.

- de l'impératif délibératif qui s'impose désormais au sein de l'ensemble des collectivités, dans le cadre des stratégies des politiques. Placé au centre du débat, le citoyen est censé jouer alors un rôle central dans ce mouvement 
de renouveau démocratique et la mise en œuvre de nouvelles procédures de concertation et d'interaction avec le citoyen reste au cœur des missions de la communication publique.

Dans quelle mesure ces problématiques contribuent-elles à la mutation du métier de communicant public ? Il semble nécessaire de revenir sur ces concepts, tant ils nourrissent les paradoxes de ce questionnement. En effet, le terme de mutation sous-entend une transformation majeure, traduisant le passage d'un état à un autre, d'un ordre à un autre, et impliquant donc une rupture; tandis que le métier défini notamment par les sociologues réfère à une organisation sociale productive ancienne, socle d'une culture professionnelle spécifique toujours d'actualité et prenant tout son sens dans cette pérennité.

Ainsi, la notion de métier renvoie à la corporation présente dès le XI ${ }^{\text {ème }}$ siècle, qui avait trois fonctions : s'assurer le monopole de la production, garantir la qualité du travail et l'honnêteté de ses membres, réglementer la formation des apprentis et l'accès à la maîtrise. L'abolition des corporations ouvre une nouvelle ère caractérisée par des relations de travail contractuelles librement consenties (XVIIIème siècle). Le taylorisme, qui se diffuse dès la fin du XIXème siècle et dans la première moitié du XXème siècle se traduit par la confiscation des savoirs des ouvriers qualifiés par les directions des entreprises ; il est alors analysé par la plupart des sociologues du travail comme une rupture par rapport à un ordre fondé sur les métiers.

Pour Florence Osty, sociologue des organisations, on assisterait depuis le processus de modernisation organisationnelle des années 80 à un retour des métiers : "la nouveauté des contextes productifs contemporains réside dans la combinaison opérée entre une rationalisation croissante et la résurgence de poches d'autonomie productive, condition de l'efficacité des univers modernes ». La notion de métier réfère à la détention de savoirs et de qualités humaines mobilisés dans l'activité productive. La notion de savoirs pratiques, notamment engagés dans des activités complexes requérant des opérations de jugement et d'arbitrage renvoie au modèle de la compétence décrit par beaucoup d'auteurs. La compétence suppose une dynamique des transformations des expériences en savoirs pratiques puis en savoirs formalisés pouvant faire l'objet d'un transfert. Pour exister, les compétences ne sont pas séparables d'une norme sociale de jugement (Osty, 2003), s’inscrivant dans une culture professionnelle et un cadre déontologique.

Pour Philippe Zarifian, Le modèle de la compétence est une forme de réponse au changement de la nature du travail avec la notion d'événement (aléas et incidents du système de production), une communication d'intercompréhension dans le travail (Zarifian, 1999). Ainsi, les compétences " sociales », « civiles », selon Zarifian, nommées par les gestionnaires les "savoir être » sont au cœur des concepts de métier et de compétence. Enfin, la dimension socialisatrice du métier met en valeur l'importance cruciale 
de l'identité (identité pour soi, identité pour autrui) qui se construit dans l'exercice de la profession (Dubar, 1991).

Le dernier paradoxe du questionnement de ce numéro de la revue réside dans le concept même de communication publique (Bessières, 2009). Concept hybride, s'il en est, car intégrant à la fois les stratégies politiques et publiques de communication, des enjeux bureaucratiques et de management public. Paradoxe qui ne fait que refléter le " processus de mutation d'un idéal normatif d'émancipation en impératif idéologique voué à la légitimation de nouvelles contraintes au service du système économique » (Honneth, 2006). Les discours participatifs qui interfèrent de manière constructive entre deux formes de communication, l'une politique et l'autre publique, sont un bon exemple de ce type de paradoxe. C'est, du reste, de cette diffraction ou « mise en morceaux » du discours participatif scindé en deux, avec d'un côté un discours politique, pragmatique, unilatéral (l'impératif) et de l'autre, un discours public, réflexif, revendiquant l'échange (participatif) que naissent les paradoxes aux effets délégitimants.

A quels changements tangibles le socle commun de compétences et l'identité professionnelle spécifique du métier de communicant public sont-ils soumis ? Peut-on évoquer le terme de mutation ou bien le changement annoncé serait-il plus une utopie, voire une idéologie qu'une réalité ? C'est ce à quoi répond ce numéro en prenant la mesure des bouleversements de l'environnement professionnel, des compétences du métier ainsi que des défis majeurs auxquels, aujourd'hui, il est confronté.

Le dossier s'ouvre sur un article de Francine Charest et Anne Gauthier de l'université de Laval. Dans une posture épistémologique forte, l'article traite du changement de "faire " des professionnels de la communication avec l'évènement du web 2.0. Au centre du processus communicationnel des " relationnistes ", l'usager est présenté comme entrant activement dans l'écosystème de l'organisation. Après une définition des médias sociaux, les auteurs pointent la transition contenue dans le délaissement progressif du paradigme linéaire de la communication au profit de nouveaux paradigmes et de nouveaux modes de gestion des relations. Un état de la situation des pratiques quotidiennes des professionnels des relations publiques est brossé, les modèles de communication interrogés.

Elizabeth Gardère de l'université de Bordeaux 4 montre comment le nouveau management public se traduit dans la gouvernance territoriale. $\mathrm{Du}$ service public à l'action publique, du cloisonnement hiérarchique à l'injonction paradoxale à l'autonomisation, toutes les figures d'une institution communicante en réorganisation nous laissent appréhender la mutation annoncée. Le nouveau management public (NMP) est arrivé et avec lui la culture de la performance et de l'évaluation, transformant le métier de communicant public. Le contexte général de révision des politiques publiques est posé. 
Olivier Le Saëc de l'université de Montpellier 3 s'attache à observer la démocratisation de l'action publique. Avec l'établissement des chartes déontologiques du communicant public, visant la reconnaissance de l'intérêt général et par là-même un certain professionnalisme, l'auteur montre la tentative d'autonomisation des communicants publics. L'observation des évolutions des missions du communicant public laisse émerger la question de la régulation des tensions entre autorités publiques et citoyens. Mutation ou leurre, interroge l'auteur en filigrane, au regard d'une utopie de la communication sous-jacente?

Hakim Akrab de l'université Stendhal de Grenoble propose une analyse sur l'appropriation des dispositifs délibératifs via internet par les établissements intercommunaux. Une enquête portant sur cinq établissements de la région Rhône-Alpes montre que le développement des procédures délibératives, souvent à visée utilitariste ou politique, participe à la mise en ouvre relativement floue d'une démocratie participative trop souvent confondue avec une démocratie de proximité, ne renforçant pas forcément l'institution publique dans sa légitimité. La mutation du métier et ses valeurs intrinsèques sont une nouvelle fois interrogées.

Valérie Lépine et Marie-France Peyrelong, respectivement de l'université Pierre Mendès France de Grenoble et de l'Ecole Supérieure en Sciences de l'Information et des Bibliothèques (Enssib) de Lyon portent un regard sur les activités et compétences qui relèvent de la communication des bibliothèques municipales ou universitaires. Leur article montre l'oscillation de la communication aux frontières des métiers de bibliothécaires et de communicants, dans un contexte spécifique de transformation des modes de management du service public. Face à une amplification des enjeux communicationnels, le paradoxe d'une communication contenue dans l'hybridation des métiers et des pratiques professionnelles semble pouvoir être levé par l'accroissement des mobilités professionnelles.

Violaine Appel et Hélène Boulanger de l'université de Loraine s'intéressent au dispositif communicationnel des universités françaises dans le contexte historique de mise en concurrence des établissements universitaires. Les auteurs mettent l'accent sur les tensions entre la logique de marque et la culture de l'organisation universitaire. Ainsi est appréhendée la question du contrôle de la circulation des informations, dans le cadre du concept d'une communication organisante.

Maryse Carmes et Yanita Andonova de l'université Paris 13 examinent la construction de politiques numériques au sein des collectivités territoriales à l'aune des processus narratifs techno-politiques contenus dans les scripts de l'e.organisation. Ces scripts déployant les politiques intranet expriment les tensions liées à des logiques contradictoires de communication descendante ou de partage des ressources et de transversalité (espaces collaboratifs). Les auteurs proposent une réflexion relative à l'influence des chefs de projet intranet 
sur le couplage TIC et innovation organisationnelle et leur confrontation à des dimensions professionnelles à la fois techniques et managériales.

Jean-Baptiste Le Corf de l'université de Paris 8 interroge le rôle des communicants municipaux dans le cadre de la mise en place d'une plate-forme numérique d'échanges de services entre habitants. Cette étude met en valeur des pratiques discursives valorisant le lien social et la contribution des usagers, considérés comme acteurs citoyens. On assiste là, à la démonstration d'une volonté politique, visant à transformer les communicants en véritables stratèges capables de susciter les pratiques contributives des habitants pour pallier en partie les insuffisances de la gestion publique locale.

Interroger la communication publique à partir de la mutation du métier réalise ainsi notre ambition de renouveler et d'approfondir les recherches en communication organisationnelle. Elles ont pour point commun de comprendre la communication publique, à partir des personnes agissant en situation professionnelle. Elles questionnent les stratégies et modèles de communication, plutôt dans leur mise en œuvre; les organisations non seulement en tant que structures mais en tant que communication instituante (organisante). Elles mettent en valeur la complexité et les paradoxes du métier, lui conférant ainsi un caractère universel dans les organisations productives : celui de l'importance des savoir être, des qualités personnelles des communicants répondant à l'hybridité des pratiques professionnelles, elle-même révélatrice de l'entrelacement de deux univers, public et politique. Aux paradoxes du questionnement répond ainsi en écho les paradoxes du métier soumis à des changements d'ampleur.

Il s'agit bien de lever le voile sur la dynamique interne de l'évolution du métier de communicant public au regard d'un contexte mouvant et d'esquisser ainsi les contours d'une mutation en train de se faire.

\section{BIBLIOGRAPHIE}

BESSIÈRES D., " La définition de la communication publique : des enjeux disciplinaires aux changements de paradigmes organisationnels ", Communication $\Xi$ Organisation, $\mathrm{n}^{\circ}$ 35, 2009.

BLONDIAUX L., SINTOMER Y., «Limpératif délibératif », Politix, Volume 15, $\mathrm{n}^{\circ}$ 57/2002, p. $17-35$.

BOLTANSKI L., CHIAPELLO È., Le nouvel esprit du capitalisme, Paris, Gallimard, 1999, 843 p.

BOLTANSKI L., THÉVENOT L., De la justification : les économies de la grandeur, Paris, Gallimard, 1991, 485 p.

BOUQUILLION P., MATTHEWS J.T., Le web collaboratif, Mutation des industries de la culture et de la communication, Grenoble, PUG, 2010,150 p. 
DUBAR C., La socialisation : construction des identités sociales et professionnelles, Paris, Armand Colin, 1991, 278 p.

HABERMAS J., Droit et démocratie. Entre faits et normes, traduit de l'allemand par ROCHLITZ R. et BOUCHINDHOMME C., Paris, Gallimard, 1997, 554 p.

HONNETH A., La société du mépris, Paris, La Découverte, 360 p.

LEMAIRE M., ZÉMOR P., (dir.), postface de J.-M. SAUVÉ, La communication publique en pratiques, Paris, La Documentation française, 2008, 442 p.

OSTY F., Le désir de métier, Engagement identité et reconnaissance au travail, Rennes, Presses universitaires de Rennes, 2003, 243 p.

WEBER M., Le savant et le politique, Paris, Union Générale d'Éditions, 1963, 186 p.

WEBER M. (publication originale, posthume, 1921), La domination légale à direction administrative bureaucratique, in TESSIER R. et TELLIER Y., Théories de l'organisation. Personnes, groupes, systèmes et environnement, chapitre 2, Québec, Les Presses de l'Université du Québec, 1991, 347 p.

WOLTON D., Indiscipliné. La communication, les hommes et la politique, Paris, Odile Jacob, 2012, $451 \mathrm{p}$.

ZARIFIAN P., Le travail et la compétence, entre puissance et contrôle, Paris, PUF, 2009, $192 \mathrm{p}$.

ZEMOR P., La communication publique. PUF, 2008, 128 p.

CAP'COM

enquête métier Cap'Com-Occurrence 2011 en accès libre sur le site

http://mediatheque.cap-com.org/enquetes/115

enquête communication interne : «Profils, métiers et organisation de la communication interne des collectivités publiques » : fichier PDF en accès libre sur le site

http://capcom.cap-com.org 
